\title{
A Comparison of Lenvatinib versus Sorafenib in the First-Line Treatment of Unresectable Hepatocellular Carcinoma: Selection Criteria to Guide Physician's Choice in a New Therapeutic Scenario
}

This article was published in the following Dove Press journal:

Journal of Hepatocellular Carcinoma

\author{
Angelo Dipasquale $\mathbb{D}^{1,2}$ \\ Arianna Marinello (D) ${ }^{1,2}$ \\ Armando Santoro $\mathbb{D}^{1,2}$ \\ 'Department of Biomedical Sciences, \\ Humanitas University, Pieve Emanuele, \\ 20090, Italy; ${ }^{2}$ IRCCS Humanitas Research \\ Hospital, Rozzano, 20089, Italy
}

\begin{abstract}
Hepatocellular carcinoma (HCC) is the fifth most common malignancy across the world. Alongside improvement in local approaches for early stages, the prognosis of patients with advanced disease remains poor. The tyrosine kinase inhibitor sorafenib was the first drug approved for advanced HCC. During the past decade, this has been extensively explored in real-life settings, such as Eastern Cooperative Oncology Group performance status 2, ChildPugh B liver function, chronic kidney disease, HIV infection, transplant recipients and the elderly. After 10 years, the multikinase inhibitor lenvatinib was approved in first-line setting. The Phase III REFLECT trial established the non-inferiority of lenvatinib compared with sorafenib in terms of overall survival, meanwhile exploratory analysis suggests a potential benefit over sorafenib for patients with HBV chronic infection and positive alpha-fetoprotein value. Experience with lenvatinib for patients not matching the REFLECT trial criteria remains promising but still retrospective. Indeed, the treatment sequence after lenvatinib still remains a crucial issue, considering that standard second-line options were tested only in patients who progressed to sorafenib. Overall, the choice between lenvatinib and sorafenib should take into account key selection criteria from randomized trials, evidence to date in special clinical situations, the physician's experience and patient's preference. Fast approval of atezolizumab plus bevacizumab as first-line treatment for advanced HCC brought an additional element in this scenario. Undoubtedly, lenvatinib and sorafenib remain available options for patients who are not suitable or those progressed to combination immunotherapy. It is conceivable that new systemic options will contribute to design a new treatment algorithm for HCC in the near future. Meanwhile, prospective studies and biomarker analysis are needed to help physicians in the choice between lenvatinib and sorafenib.
\end{abstract}

Keywords: hepatocellular carcinoma, sorafenib, lenvatinib

\section{Introduction}

Hepatocellular carcinoma (HCC) is the fifth most common malignancy and the second most frequent cause of cancer-related deaths worldwide, with an incidence of 841,080 cases and an absolute number of deaths of 781,631 in $2018 .{ }^{1,2}$ Its incidence has increased in the past decades in western regions, while in other countries such as Japan it has started to decline. ${ }^{1,2}$

Approximately $90 \%$ of HCCs are associated with a known underlying etiology, most frequently chronic hepatitis induced by hepatitis virus $\mathrm{B}$ (HBV) and C (HCV) in approximately $54 \%$ and $31 \%$ of cases, respectively. ${ }^{1-3}$ Alcohol intake and
Correspondence: Armando Santoro IRCCS Humanitas Research Hospital, Via Manzoni 56, Rozzano, 20089, Italy

Tel +3928224 4080

Fax +39282244592

Email armando.santoro@humanitas.it
Journal of Hepatocellular Carcinoma 2021:8 24I-25I 
aflatoxin exposure are responsible for $15 \%$ of cases. ${ }^{1-3}$ In Africa and east Asia, the predominant etiology is $\mathrm{HBV}$ infection $(60 \%)$, in contrast to only $20 \%$ of cases in the western countries, where $\mathrm{HCV}$ is the most important risk factor. ${ }^{3}$

Moreover, all etiologic forms of cirrhosis may be complicated by tumor formation and approximately one-third of cirrhotic patients will develop HCC during their lifetime. $^{1-3}$ Two distinct and potentially lethal diseases, cancer and cirrhosis, usually coexist in patients with HCC and hepatic function evaluation is crucial. Liver function is most commonly categorized according to the Child-Pugh (CP) score, which considers clinical and biochemical items and has a strong prognostic value. ${ }^{4,5}$ Liver function is included in integrated functional and staging scores, such as the Barcelona Clinic Liver Cancer (BCLC) model, which define resectable (BCLC stage A), intermediate (BCLC stage B) and advanced (BCLC stage C) disease according to clinical, biochemical and radiological features. ${ }^{6,7}$ While early and intermediate HCC may benefit from a local approach (surgery or interventional radiology) or orthotopic liver transplantation (OLT) in selected cases, treatment options for advanced HCC are still limited. ${ }^{8-10}$

In recent years, it has become clear that $\mathrm{HCC}$ represents a distinct disease even for tumor response evaluation. In 2000, a panel of experts convened by the European Association for the Study of the Liver (EASL) amended the response criteria to consider tumor necrosis induced by treatment. $^{11,12}$ The estimated reduction in the viable tumor area in contrast-enhanced radiologic imaging was considered as the optimal method to assess treatment response. ${ }^{11,12}$ These amendments to Response Evaluation Criteria in Solid Tumours (RECIST) criteria were defined as modified RECIST assessment (mRECIST) for HCC and were adopted for the evaluation of response to treatment. ${ }^{11,12}$

Despite the improvement in prognosis for early stages, survival for patients with advanced disease remains poor. The tyrosine kinase inhibitors (TKIs) sorafenib and lenvatinib have been the up-front treatment for advanced HCC in the past decade. In this article, we will briefly review the available evidence for sorafenib and lenvatinib in clinical trial populations and even in real-world settings, with a focus on patients not matching phase III study inclusion criteria and special populations. Subsequently, we will explore several scenarios in which sorafenib could be preferred over lenvatinib and vice versa. Finally, we will discuss the still actual role of TKIs in the immunotherapy era, where the anti-programmed death ligand 1 (PD-L1) agent atezolizumab plus bevacizumab and other combinations are changing the first-line treatment scenario.

\section{Outlook: First-Line Sorafenib and Lenvatinib \\ Sorafenib}

Sorafenib is a TKI targeting the RAF/MEK/ERK axis of the RAS cascade signal, vascular endothelial growth factor receptors (VEGFRs) 1-3 and the platelet-derived growth factor receptor $\beta$ (PDGFR- $\beta$ ). ${ }^{13}$

In 2007, the Phase III, double-blind, randomized trial SHARP explored sorafenib as first-line treatment for advanced HCC. ${ }^{14}$ A total of 602 patients were randomized to receive either sorafenib $400 \mathrm{mg}$ twice daily or placebo. ${ }^{14}$ Patients characteristics included western ethnicity, Eastern Cooperative Oncology Group (ECOG) performance status (PS) ranging from $0(54 \%)$ to $2(8 \%)$ and a preserved liver function (CP class A for $95 \%$ of patients). ${ }^{14} \mathrm{HCV}$ was the most common cause of chronic hepatitis (29\%), macroscopic vascular invasion was present in $38 \%$ of cases and $53 \%$ of patients presented an extrahepatic spread. ${ }^{14}$ The study met the primary endpoint of median overall survival (mOS; 10.7 months vs 7.9 months, $\mathrm{p}<0.001$ ), meanwhile the co-primary endpoint of time to symptomatic progression (defined as a decrease in $\geq 4$ points during treatment on the FHSI8 questionnaire) showed no difference between the two arms (4.1 vs 4.9 months, $\mathrm{p}=0.77) .{ }^{14}$ Secondary endpoints included disease control rate according to RECIST 1.0 criteria ( $2 \%$ of partial response $[\mathrm{PR}]$ and $71 \%$ of stable disease [SD]) and time to radiological progression (TTRP; 5.5 vs 2.8 months, $\mathrm{p}<0.001) .{ }^{14}$ The benefit of sorafenib was seen across all subgroups of patients. ${ }^{14}$ Overall, $76 \%$ of patients received more than $80 \%$ of the planned dose. ${ }^{14}$ In the experimental group, the more common adverse events (AEs) were diarrhea (39\%) and hand-foot skin reaction (HFSR; 21.2\%). ${ }^{14}$ The discontinuation rate for toxicity was similar between the two cohorts (38\% vs 37\%). ${ }^{14}$

The phase III ASIA-PACIFIC study explored the efficacy of sorafenib even in the eastern population. ${ }^{15}$ In this study, 226 patients were randomized to receive either sorafenib or placebo. ${ }^{15}$ Compared to SHARP, the ASIAPACIFIC trial encompassed a population characterized by unfavorable prognosis, with a higher rate of ECOG PS 1 (69.3\%), extrahepatic spread (68.7\%), chronic HBV infection $(70.7 \%)$ and alpha-fetoprotein (AFP) value superior to 
the upper limit of normal (77.3\%). ${ }^{15}$ No primary endpoint was defined for the study but sorafenib improved mOS (6.5 vs 4.2 months, $\mathrm{p}=0.014){ }^{15}$ Median progression-free survival (mPFS), objective response rate (ORR), time to symptomatic progression and safety profile followed the SHARP trial results, except for a higher incidence of HFSR (45\% vs $21.2 \%) .{ }^{15}$

A meta-analysis of these two phase III studies confirmed the benefit of sorafenib across all subgroups of patients, in particular when intrahepatic disease, chronic $\mathrm{HCV}$ infection and a neutrophil to lymphocyte ratio equal to or under the median value were present. ${ }^{16}$

In the last decade, sorafenib was extensively explored in the real-life setting and the management of its AEs has strongly improved in recent years. An interesting retrospective analysis including 188 patients divided in two period cohorts (2007-2012 vs 2013-2017) found a statistically significant difference in terms of duration of treatment (8 vs 12 months), mOS (8 vs 12 months) and mOS for patients achieving disease control at 8 weeks (13 vs 23 months). ${ }^{17}$ This finding suggested an improvement in the management of skin toxicity in recent times, allowing patients to stay longer in treatment and potentially improving outcome. ${ }^{17}$ Other studies analyzed the activity and safety of sorafenib in patients not matching SHARP and ASIA-PACIFIC key inclusion criteria. ${ }^{18-29}$

The GIDEON study prospectively tested sorafenib in 3371 patients, including CP class B $(25 \%) .{ }^{18}$ Compared with patients with preserved liver function ( $\mathrm{CP}$ class $\mathrm{A}$ ), the entire $\mathrm{CP}$ class $\mathrm{B}$ cohort showed a worse mOS, with no difference in the AEs frequency. ${ }^{18}$ In opposition to CP B8/ 9, patients classified as CP B7 (with no ascites, encephalopathy or coagulation disorders) appeared as the subgroup taking the most benefit from treatment with sorafenib, with higher mOS (4.8/3.7 months vs 6.2 months) and lower rate of grade $3-4$ AEs $(68 / 69 \%$ vs $54 \%){ }^{18}$

Joining the therapeutic armamentarium of metastatic renal cell carcinoma, sorafenib was also tested in patients with chronic kidney disease (CKD). ${ }^{19-21}$ A pharmacokinetic analysis showed no difference in plasma levels between subjects affected by renal insufficiency and healthy ones. ${ }^{19}$ The observation was confirmed in a retrospective analysis conducted on 14 patients affected by metastatic renal cell carcinoma treated with sorafenib and with a serum creatinine clearance $\leq 60 \mathrm{~mL} / \mathrm{min} .{ }^{20}$ Compared to 18 cases with normal renal function, no difference in terms of ORR, TTRP and mOS was detected, despite a higher rate of diarrhea (33\% vs $57 \%$ ), dose interruption ( $28 \%$ vs $57 \%$ ) and dose reduction
$(22 \%$ vs $43 \%) .{ }^{20}$ Interestingly, a single-center experience showed efficacy and safety of sorafenib in two patients undergoing hemodialysis, except for the development of hypertension. ${ }^{21}$

Patients affected by HCC with concomitant HIV infection merit a special attention, considering the potential drug-drug interaction between the TKI and highly active anti-retroviral therapy. ${ }^{22-25}$ Both protease inhibitors and sorafenib are metabolized by cytochrome CYP3A4, but no change was observed for TKI plasma levels in healthy subjects taking the CYP3A4 inhibitor ketoconazole. ${ }^{22}$ On the other hand, non-nucleotide reverse transcriptase inhibitors are CYP3A4 inducers and could impact on sorafenib bioavailability, as already demonstrated for the TKI in patients taking the inducer drug rifampicin. ${ }^{23}$ The combination of a protease inhibitor plus two non-nucleotide reverse transcriptase inhibitors appears as the best choice for the highly active anti-retroviral therapy regimen during treatment with sorafenib. Single experiences supported this approach alongside the efficacy and safety of sorafenib in HIV-infected patients. ${ }^{24,25}$

It is known that $10-15 \%$ of patients matching the Milan criteria and undergoing OLT will experience extrahepatic relapse requiring systemic therapy. ${ }^{26,27}$ A retrospective analysis on nine patients relapsing after OLT confirmed the activity of sorafenib (one complete response, four $\mathrm{SD}$, three progressive disease [PD], one case not evaluable). ${ }^{26}$ Even with a step-up approach to the full schedule, permanent dose reduction was required in six patients for HFSR (grade 3-4 in two cases) and/or diarrhea (grade 3 in one case). ${ }^{26}$ Similar results came from a Korean experience including 13 patients receiving sorafenib at recurrence after living-donor liver transplantation. ${ }^{27}$ Sorafenib appeared active (six SD, four PD, three cases not evaluable) and AEs were observed across the entire cohort, including diarrhea (81\%), fatigue (73\%), nausea (64\%), HFSR (55\%), hair loss $(55 \%)$ and weight loss $(55 \%){ }^{27}$

Finally, elderly patients are a special subset usually excluded from clinical trials. A recent prospective study confirmed the benefit of sorafenib in 792 elderly people (age $\geq 75$ years) when compared to a younger cohort $(<75$ years), with no difference in mOS (7.3 vs 7.2 months, $\mathrm{p}=0.97) .{ }^{28}$ Interestingly, a dose-escalation strategy to the full schedule showed no correlation with clinical outcome. ${ }^{28}$ Safety profile was similar between the two groups, except for moderate-to-severe anorexia (14\% vs $7.2 \%)$ and cutaneous rash $(6.3 \%$ vs $3.1 \%) .{ }^{28}$ However, 
Table I Failed Multicenter, Randomized, Phase III Trials in Patients with Untreated Advanced HCC

\begin{tabular}{|c|c|c|c|}
\hline Randomized Phase III Trials & Target & Patients (n) & Results (OS) \\
\hline Sunitinib vs sorafenib ${ }^{55}$ & VEGFR, PDGFR, others & 1074 & 7.9 vs 10.2 months; HR I.30 (95\% Cl: I.। $3-I .50), p=0.00 ।$ \\
\hline Brivanib vs sorafenib (BRISK-FL) ${ }^{56}$ & FGFR, VEGFR & 1155 & 9.5 vs 9.9 months; HR I.07 (95\% Cl: $0.94-I .23), p=0.3$ I \\
\hline Sorafenib \pm erlotinib $(\mathrm{SEARCH})^{57}$ & EGFR & 731 & 9.5 vs 8.5 months; HR 0.93 (95\% Cl: $0.78-I .1 \mathrm{I}), p=0.4 \mathrm{I}$ \\
\hline Linifanib vs sorafenib ${ }^{58}$ & VEGFR, PDGFR, others & 1035 & 9.1 vs 9.8 months; HR I.05 (95\% Cl: 0.9-I.22), $p=N R$ \\
\hline Sorafenib \pm doxorubicin ${ }^{59}$ & - & 356 & 9.3 vs 9.4 months; HR I.05 ( $95 \%$ Cl: $0.83-I .3$ I), $p=0.68$ \\
\hline
\end{tabular}

Abbreviations: EGFR, epidermal growth factor receptor; FGFR, fibroblast growth factor receptor; HR, hazard ratio; OS, overall survival; PDGFR, platelet-derived growth factor receptor; VEGFR, vascular endothelial growth factor receptor.

higher discontinuation rate for AEs was noted in the elderly population $(27 \%$ vs $21.6 \%){ }^{28}$

In Table 1, we report a number of negative trials conducted across the years, showing that no molecule improved survival in the first-line setting. Sorafenib remained the only option for patients with advanced disease for 11 years until lenvatinib, another multi-kinase inhibitor, was approved in first-line setting in 2018.

\section{Lenvatinib}

Lenvatinib is a TKI targeting VEGFR 1-3, PDGFR- $\alpha$, fibroblast growth factor receptor 1-4 (FGFR 1-4), KIT and rearrangement during transfection (RET) ${ }^{29}$

Through the inhibition of VEGFR and FGFR pathways, lenvatinib activity is characterized by a more pleiotropic effect when compared to sorafenib. ${ }^{29-32}$ Intriguingly, the two TKIs shared comparable anti-tumor activity in immunodeficient mouses but not in immunocompetent ones, suggesting a role for the immune system in increasing lenvatinib cytotoxic effect. ${ }^{30}$ In fact, preclinical experiences confirmed its action on the tumor micro-environment, inducing a CD8+ $\mathrm{T}$ cell infiltration and decreasing the number of tumorassociated macrophage. ${ }^{31,32}$

The phase III, open-label, randomized REFLECT trial was conducted across eastern and western populations to demonstrate the non-inferiority of lenvatinib in comparison to sorafenib in terms of survival. ${ }^{33}$ The target population was slightly different from the one in SHARP and ASIA-PACIFIC trials because of the exclusion of ECOG PS 2 patients and the presence of low disease burden $(50 \%$ or more liver occupation and/or bile duct or main portal vein invasion were exclusion criteria). ${ }^{33}$ A total of 954 patients were randomized to receive either lenvatinib or sorafenib and the non-inferiority margin for OS was set at a hazard ratio (HR) of $1.08 .{ }^{33}$ Baseline characteristics were well balanced between the two arms with the exception of HCV chronic infection and baseline AFP $<200 \mathrm{ng} / \mathrm{mL}$, more frequent in the Sorafenib group (26\% and $39 \%$ of cases, respectively). ${ }^{33}$ Lenvatinib met the primary endpoint of non-inferior mOS (13.6 vs 12.7 months, HR: 0.92; 95\% CI: 0.79-1.06) and outperformed sorafenib across all secondary endpoints, including mPFS ( 7.4 vs 3.7 months, $p<0.0001$ ), TTRP ( 8.9 vs 3.7 months, $\mathrm{p}<0.0001)$ and ORR according to mRECIST criteria $(24.1 \%$ vs $9.2 \%, \mathrm{p}<0.0001) .{ }^{33}$ An independent imaging review showed an ORR of $40.6 \%$ according to mRECIST criteria. ${ }^{33}$ When OS results were adjusted for AFP levels, lenvatinib appeared superior to sorafenib (HR: 0.85; 95\% CI: 0.73-0.99). ${ }^{33}$ During treatment, both groups showed a decrease in quality of life according to EORTC QLQ-C30 and HCC-18 questionnaires, although a delay in deterioration for different items (role function, diarrhea, pain, body image, nutrition, fatigue and sexual function) was seen for lenvatinib. ${ }^{33}$ In the lenvatinib arm, the most common AEs were hypertension (42\%), diarrhea (39\%), anorexia (34\%), weight loss (31\%) and proteinuria (25\%), with higher rate of severe AEs $(57 \%$ vs $49 \%$ in the sorafenib cohort). ${ }^{33}$ Dose reduction and discontinuation rate were similar between the two arms (37\% vs $38 \%$ and $9 \%$ vs $7 \%$, respectively) but longer treatment duration was seen for lenvatinib (5.7 vs 3.7 months). ${ }^{33}$ The OS in REFLECT trial was the longest to be reported at that time in first-line setting. Both the selection of patients with good prognosis and the high frequency of postprogression treatments (in particular in western countries, $28 \%$ for the lenvatinib arm and $45 \%$ for the sorafenib arm) could have contributed to the excellent outcome. ${ }^{33}$ The longer treatment duration with lenvatinib was explained, in part, by a lower rate of symptomatic AEs, such as HFSR and diarrhea, improving compliance. ${ }^{33}$ However, the open-label design (with the awareness in taking the control drug) could have led to premature interruption of 
sorafenib, potentially justifying the shorter treatment duration and the inferior outcome in the opposite arm. ${ }^{33}$

Biomarker analysis demonstrated a correlation between higher plasmatic levels of FGF-2 and angiopoietin 2 and longer OS during treatment with lenvatinib. ${ }^{34}$

Recently, a series of experiences tested the efficacy and safety of lenvatinib outside REFLECT trial criteria. A retrospective trial explored lenvatinib in 152 patients in different clinical scenarios, such as patients pretreated with sorafenib and a high disease burden (main portal vein and/or bile duct invasion or $\geq 50 \%$ liver occupation). ${ }^{35}$ The mPFS for patients with preserved liver function receiving lenvatinib in the- first and second-line setting was similar (5.2 vs 4.8 months, $\mathrm{p}=0.933$ ). ${ }^{35}$ In the subset characterized by high intrahepatic disease burden ( 27 cases), the ORR according to mRECIST criteria was $41 \%$, as the one in REFLECT trial. ${ }^{35}$ However, when considering patients classified as CP A, a difference in mOS between high and low disease burden was seen (6.5 vs 14.2 months, $\mathrm{p}=0.014) .{ }^{35}$ The trial included $13 \%$ of patients with impaired liver function (CP B). ${ }^{35}$ Compared to $\mathrm{CP}$ A, CP $\mathrm{B}$ patients with a score $\geq 7$ presented higher rate of dose interruption (50\%) and liver-related AEs, such as aspartate transaminase (AST) elevation (45\%), hepatic encephalopathy $(30 \%)$ and bilirubin elevation $(25 \%){ }^{35}$

Another retrospective study on 41 patients explored lenvatinib in a cohort not matching REFLECT trial criteria (56.1\% of the overall population). ${ }^{36}$ Again, the ORR according to mRECIST criteria did not statistically differ between patients matching and not matching REFLECT study population $(61.1 \%$ vs $60.9 \%) .{ }^{36}$ Even in this case, CP B patients with a score $\geq 6$ appeared a frail subgroup, showing higher rate of liver function deterioration $(63.2 \%$ vs $27.3 \%$ in the CP A group). ${ }^{36}$ The same research group conducted a similar analysis on 64 patients not matching the REFLECT trial criteria in a larger retrospective cohort (105 patients). ${ }^{37}$ No statistically significant difference in ORR according to mRECIST and mPFS was observed between patients matching or not REFLECT trial population $(61.5 \%$ and 10.3 months vs $48.3 \%$ and 9.08 months). ${ }^{37}$

Alongside these promising results, a retrospective Korean experience on 92 patients suggested a less enthusiastic picture, showing lower mPFS and mOS (4 months and 6 months, respectively) for patients with high disease burden $\left(28\right.$ cases) ${ }^{38}$ Even in this case, the CP B subset showed statistically lower ORR, mPFS and $\mathrm{mOS}(5.6 \%$, 2.6 months, 5.3 months, respectively) when compared to patients with preserved liver function $(\mathrm{CP} A) \cdot{ }^{38}$ Finally, a retrospective analysis on 181 patients treated with firstline lenvatinib (126 classified as CP A and 55 classified as CP B) found a significant association between CP score, response rate and survival, suggesting a worse outcome when impaired liver function was present. ${ }^{39}$

To date, no data or reports for lenvatinib in CKD, HIV infection and relapse after OLT are available. A retrospective analysis of 100 cases confirmed the efficacy and safety of lenvatinib in the elderly population ( $>75$ years) when compared to a younger cohort (median age of 69 years), with no difference in survival and rate of AEs between the two groups. ${ }^{40}$

In the REFLECT trial, lenvatinib showed an impressive ORR according to mRECIST criteria $(41 \%$ in the independent imaging review), higher than the one reported with atezolizumab plus bevacizumab (33.2\% according to mRECIST) and similar to the one observed after transarterial chemoembolization in intermediate $\mathrm{HCC}(40 \%$ according to mRECIST). ${ }^{10,11,33,41}$ In the non-advanced setting, lenvatinib as a bridge to local treatment and OLT certainly requires further investigations in the near future. For example, a case series reported that transarterial chemoembolization followed by lenvatinib induced downstaging from BCLC B to A stage in three patients, allowing surgery or ablative therapy and with no disease recurrence after 6 months. ${ }^{42}$

\section{How to Choose Between Sorafenib and Lenvatinib?}

How to choose between sorafenib and lenvatinib still remains a matter of debate. Actual international guidelines do not indicate which drug should be considered first and in which situation.

As shown in Figure 1, there is no alternative to sorafenib for patients not matching REFLECT trial criteria, such as disease with $\geq 50 \%$ liver occupation and/or bile duct or main portal vein invasion. ${ }^{14,15,33}$ In this setting, no high-quality evidence for lenvatinib exists and available data still remain retrospective and sometimes contrasting. ${ }^{35-39}$ Sorafenib appears safe in CP class B patients matching a score $\leq 7$, for which instead lenvatinib showed increased toxicity and a higher rate of liver function deterioration, potentially precluding further treatments. ${ }^{35-39}$ Several reports suggest that sorafenib is active and safe in special population, such as transplant recipients and patients with CKD and HIV infection. ${ }^{19-27}$ 
Lenvatinib a better option if:

- PS ECOG 0-1

- < $50 \%$ liver occupation.

- no bile duct/main portal vein invasion.

- HBV chronic infection

- AFP > $200 \mathrm{ng} / \mathrm{mL}$

- Child Pug A

$-<45$ y/o

- lower costs

- (downstaging)
Sorafenib a better option if:

PS ECOG

$>50 \%$ liver occupation.

- bile duct/main portal vein invasion

- HCV chronic infection.

- AFP $<200$ ng/mL

- Child Pug B(7)

$\geq 75 \mathrm{y} / \mathrm{o}$

- higher costs

- transplant recipients, HIV infection, CKD

Figure I Criteria to choose between lenvatinib and sorafenib in patients matching REFLECT trial criteria.

Abbreviations: AFP, alpha-fetoprotein; CKD, chronic kidney disease; HBV, hepatitis Bchronic Infection; HCV, hepatitis Cchronic infection; PS, performance status.

Sorafenib should be reserved for ECOG PS 2 patients, including in both SHARP and ASIA-PACIFIC trials. ${ }^{14,15}$ Finally, the efficacy and safety of sorafenib were prospectively validated in an elderly cohort $(\geq 75$ years), for which instead evidence for lenvatinib remains retrospective. However, the spectrum of asymptomatic AEs makes the second one an attractive choice in this frail population. ${ }^{28,33,40}$ When starting sorafenib in special populations, a dose-escalation strategy to test tolerability should be recommended.

Lenvatinib could represent the best choice for patients matching REFLECT trial criteria and when negative prognostic factors, such as chronic HBV infection and AFP positive value, are present. ${ }^{33}$ Despite no outcome difference in the overall population was seen, post hoc analysis showed less benefit from sorafenib in patients with HBV chronic infection. ${ }^{33}$ Indeed, lenvatinib outperformed sorafenib in terms of OS when the statistical analysis was adjusted for a positive AFP value. ${ }^{33}$ Younger patients in the prime of professional and social life can benefit most from the spectrum of asymptomatic AEs, such as hypertension and proteinuria, potentially contributing to quality of life preservation. ${ }^{33}$ Moreover, lenvatinib appeared more convenient than sorafenib. A Japanese cost-utility analysis on the AFP-adjusted population of REFLECT trial confirms that lenvatinib is more effective and cheaper in firstline setting. ${ }^{43}$ Similar analysis performed in western countries and including the overall population of REFLECT study confirmed these results. ${ }^{44,45}$
The treatment sequence after lenvatinib still remains a crucial issue. Standard second-line options, such as regorafenib, cabozantinib, ramucirumab and ipilimumab plus nivolumab, were tested only in patients with prior exposure to sorafenib. ${ }^{46-49}$ The same performance in patients progressed to lenvatinib cannot be inferred with confidence, also considering the different mechanism of action. $^{29-32}$

\section{How the New Therapeutic Scenario May Change the Choice Between Sorafenib and Lenvatinib?}

After deceiving results in randomized trials of nivolumab compared to sorafenib as up-front treatment and pembrolizumab over placebo in the second-line setting, several studies have been conducted to overcome primary and secondary resistance to immune checkpoint inhibitors. ${ }^{50,51}$ Immunotherapy burst the first-line scene with the phase III, randomized trial IMbrave150 comparing atezolizumab plus bevacizumab over sorafenib. ${ }^{41}$ Eligible patients had an ECOG PS of 0 or 1 and good liver function (CP class A). ${ }^{41}$ A history of autoimmune disease and untreated esophageal varices were key exclusion criteria. ${ }^{41}$ Atezolizumab plus bevacizumab was superior to sorafenib in terms of mPFS (6.8 vs 4.3 months, HR: $0.59, \mathrm{p}<0.001$ ) and mOS (not reached vs 13.2 months, HR: $0.58, \mathrm{p}<0.0001) .{ }^{41}$ The ORR according to mRECIST criteria was significantly higher in the experimental arm (33\% vs $13 \%)$. ${ }^{41}$ As for safety, $15 \%$ of patients discontinued the combination therapy for AEs (vs 
$10 \%$ of patients taking single-agent TKI).$^{41}$ Upper gastrointestinal bleeding was observed in $6.4 \%$ of cases for the experimental treatment (vs $4.5 \%$ in the sorafenib group), even after a careful evaluation for esophageal varices before enrollment. ${ }^{41}$ Considering this impressive result, the US Food and Drug Administration (FDA) gave fast approval to atezolizumab plus bevacizumab as first-line treatment of advanced HCC in May 2020, now considered the new standard of care in the advanced setting. ${ }^{41}$

Despite the terrific progress in treatment landscape of liver cancer, several considerations should be made before leaving TKIs behind. First, key exclusion criteria of IMbrave150 included main portal vein invasion, untreated esophageal varices, autoimmune disorders, chronic immunosuppressive therapy and very old age (median age for the combination was 64 years). ${ }^{41}$ Secondly, severe gastrointestinal bleeding was observed in $6.4 \%$ of cases and so bevacizumab should be proposed with caution during anticoagulant/antiplatelet treatment, if platelet count is very low or in patients affected by active inflammatory bowel diseases. ${ }^{41,52}$ Thirdly, the efficacy and safety of the combination therapy were tested only in patients with a preserved liver function (CP A). ${ }^{41}$ Finally, both atezolizumab and bevacizumab are high-cost drugs that will not be immediately available worldwide. Taking into account these observations, it is clear that TKIs remain the firstline option for a relevant subset of patients not suitable for atezolizumab plus bevacizumab.

Alongside the first-line setting, a different scenario to be dissected is the role of sorafenib and lenvatinib in patients progressed to atezolizumab plus bevacizumab. To date, no studies explored the two drugs in second-line setting and their activity after a VEGF inhibitor such as bevacizumab is not known. All SHARP, ASIA-PACIFIC and REFLECT trials included only VEGFR-naïve patients. ${ }^{14,15,33}$ On the other hand, regorafenib and cabozantinib showed efficacy in patients progressed to a prior VEGFR inhibitor and could be also considered in this setting. ${ }^{46,47}$ Indeed, the CELESTIAL trial included even a small number of patients progressed to anti-PD-(L) 1 immune checkpoint inhibitors (3\%), suggesting a retained activity of cabozantinib after immunotherapy failure. ${ }^{47,53}$ It follows that no standard second-line treatment after atezolizumab plus bevacizumab exists. Both sorafenib/lenvatinib and, at least in theory, also cabozantinib appear reasonable options, pending for large prospective studies. Figure 2 shows a potential treatment algorithm for advanced $\mathrm{HCC}$ in immunotherapeutic era.
Alongside the impressive result of IMbrave150, encouraging results in term of efficacy and safety are coming from other systemic treatments. For instance, the association of pembrolizumab plus lenvatinib as first-line therapy was recently investigated in a Phase I single-arm trial including 100 patients affected by advanced HCC. ${ }^{54}$ The association appears very intriguing, considering the ability of lenvatinib to induce several changes in tumor immune milieu. ${ }^{29-32}$ Key inclusion criteria were preserved liver function (CP class A) and ECOG PS $0-1 .^{54}$ Exclusion criteria included bile duct and/or main portal vein invasion and liver occupation $>50 \%$, as in REFLECT trial. ${ }^{33,54}$ After a median follow-up of 10.6 months, ORR according to mRECIST criteria was $46 \%$ (including $11 \%$ of $\mathrm{CR}$ ), mPFS was 9.3 months and mOS was 22 months. $^{54}$ Severe AEs occurred in $67 \%$ of patients, with hypertension as the most common $(17 \%) .{ }^{54}$ No increase in number of bleeding events was observed. ${ }^{54}$ In conclusion, pembrolizumab plus lenvatinib appear as a new promising therapeutic option for advanced HCC to be explored in a larger phase III trial.

\section{Conclusion and Future Perspectives}

Although atezolizumab plus bevacizumab lead to an impressive shift in treatment paradigm of advanced $\mathrm{HCC}$, both sorafenib and lenvatinib still remain the up-front therapeutic option for selected patients not suitable for immunotherapy or at high risk of bleeding. Furthermore, both TKIs are available for patients progressed to first-line immunotherapy combination.

The choice between sorafenib and lenvatinib should be taken considering the clinical setting, key inclusion/exclusion criteria of randomized trials, evidence coming from retrospective and prospective studies on special populations, safety profile and patient's preferences. Moreover, it is reasonable to assume that new systemic approaches will further contribute to diversify the therapeutic algorithm of advanced HCC in the near future.

In our opinion, conducting prospective studies should be strongly pursued, in order to explore sorafenib and lenvatinib in real-word scenarios either as first-line treatments or as steps of a new therapy sequence for HCC. A pragmatic trial design, including different cohorts simultaneously, such as ideal populations and also real-world patients, could help to clarify the role of TKIs in everyday clinical practice. Indeed, biomarker analysis should be implemented, looking for easy-to-use predictive and prognostic variables which may improve 


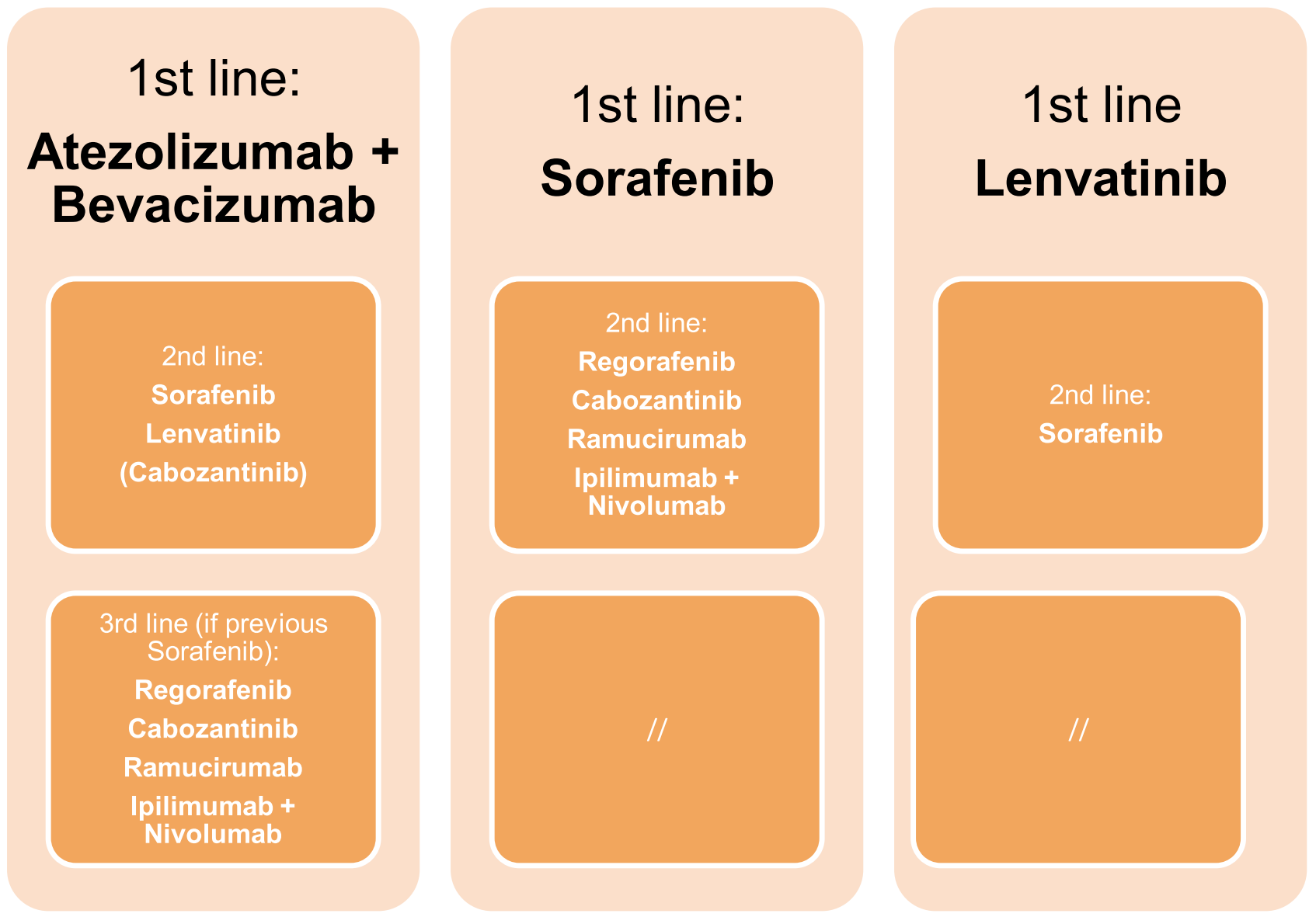

Figure 2 Proposed therapeutic algorithm for advanced HCC in immunotherapeutic era.

the choice between lenvatinib or sorafenib. This could be of paramount importance even in immunotherapy era, in order to identify patients with primary resistance to anti-PD-L1 treatment. Finally, exploring the activity of TKIs after up-front immunotherapy combination should be a high priority.

Future studies will contribute to dissect the best treatment sequence among the available systemic options, helping physicians in selecting the right drug for the right patient.

\section{Abbreviations}

AEs, Adverse events; AFP, Alpha-fetoprotein; BCLC, Barcelona Clinic Liver Cancer; CP, Child-Pugh; EASL, European Association for the Study of the Liver; ECOG, Eastern Cooperative Oncology Group; FGFR, Fibroblastgrowth factor receptor; HBV, Hepatitis virus B; HCC, Hepatocellular carcinoma; HCV, Hepatitis virus C; HFSR, Hand-foot skin reaction; HR, Hazard ratio; mOS, Median overall survival; mPFS, Median progression-free survival; mRECIST, Modified Response
Evaluation Criteria in Solid Tumours; OLT, orthotopic liver transplantation; ORR, Objective response rate; PDGFR- $\alpha / \beta$, Platelet-derived growth factor receptor $\alpha / \beta$; PD-L1, Programmed death ligand 1; PR, Partial response; PS, Performance status; RECIST, Response Evaluation Criteria in Solid Tumours; RET, rearrangement during transfection; SD, Stable disease; TKI, Tyrosine kinase inhibitor; TTRP, Time to radiological progression; VEGFR, Vascular endothelial growth factor receptor.

\section{Data Sharing Statement}

None required.

\section{Ethics Approval and Informed Consent}

None required.

\section{Consent for Publication}

Obtained. 


\section{Acknowledgments}

Editorial assistance was provided by Aashni Shah (Polistudium SRL, Milan, Italy). This assistance was supported by internal funds.

\section{Funding}

The authors have not declared a specific grant for this research from any funding agency in the public, commercial or not-for-profit sectors.

\section{Disclosure}

The authors report no conflicts of interest in this work.

\section{References}

1. Globocan. The IARC Global Cancer Observatory. Available from: http://globocan.iarc.fr/old/FactSheets/cancers/liver-new.asp.

Accessed March 9, 2021.

2. Petrick JL, Kelly SP, Altekruse SF, McGlynn KA, Rosenberg PS. Future of hepatocellular carcinoma incidence in the United States forecast through 2030. J Clin Oncol. 2016;34(15):1787-1794. doi:10.1200/JCO.2015.64.7412

3. Akinyemiju T, Abera S, Ahmed M, et al. The burden of primary liver cancer and underlying etiologies from 1990 to 2015 at the global, regional, and national level. JAMA Oncol. 2017;3:1683-16914. doi:10.1001/jamaoncol.2017.3055

4. Pugh RN, Murray-Lyon IM, Dawson JL, Pietroni MC, Williams R. Transection of the oesophagus for bleeding oesophageal varices. $\mathrm{Br}$ J Surg. 1973;60(8):646-649. doi:10.1002/bjs.1800600817

5. Kudo M, Chung H, Osaki Y. Prognostic staging system for hepatocellular carcinoma (CLIP score): its value and limitations, and a proposal for a new staging system, the Japan Integrated Staging Score (JIS score). J Gastroenterol. 2003;38(3):207-215. doi:10.1007/s005350300038

6. European Association for the Study of the Liver. EASL Clinical Practice Guidelines: management of hepatocellular carcinoma. J Hepatol. 2018;69:182-236.

7. Forner A, Reig M, Bruix J. Hepatocellular carcinoma. Lancet. 2018;391(10127):1301-1314. doi:10.1016/S0140-6736(18)30010-2

8. Llovet JM, Fuster J, Bruix J. Intention-to-treat analysis of surgical treatment for early hepatocellular carcinoma: resection vs. transplantation. Hepatology. 1999;30:1434-1440. doi:10.1002/hep.510300629

9. Ishizawa T, Hasegawa K, Aoki T, et al. Neither multiple tumors nor portal hypertension are surgical contraindications for hepatocellular carcinoma. Gastroenterology. 2008;134:1908-1916. doi:10.1053/j. gastro.2008.02.091

10. Berzigotti A, Reig M, Abraldes JG, Bosch J, Bruix J. Portal hypertension and the outcome of surgery for hepatocellular carcinoma in compensated cirrhosis: a systematic review and meta-analysis. Hepatology. 2015;61(2):526-536. doi:10.1002/hep.27431

11. Bruix J, Sherman M, Llovet JM, et al. Clinical management of hepatocellular carcinoma. Conclusions of the Barcelona-2000 EASL conference. European Association for the Study of the Liver. J Hepatol. 2001;35(3):421-430. doi:10.1016/S0168-8278(01)00130-1

12. Bruix J, Sherman M. Practice Guidelines Committee, American Association for the Study of Liver Diseases. Management of hepatocellular carcinoma. Hepatology. 2005;42(5):1208-1236.

13. Wilhelm SM, Carter C, Tang L, et al. BAY 43-9006 exhibits broad spectrum oral antitumor activity and targets the RAF/MEK/ERK pathway and receptor tyrosine kinases involved in tumor progression and angiogenesis. Cancer Res. 2004;64(19):7099-7109. doi:10.1158/ 0008-5472.CAN-04-1443
14. Llovet JM, Ricci S, Mazzaferro V, et al. Sorafenib in advanced hepatocellular carcinoma. $N$ Engl J Med. 2008;359:378-390. doi:10. 1056/NEJMoa0708857

15. Cheng A-L, Kang Y-K, Chen Z, et al. Efficacy and safety of sorafenib in patients in the Asia-Pacific region with advanced hepatocellular carcinoma: a phase III randomised, double-blind, placebo-controlled trial. Lancet Oncol. 2009;10:25-34. doi:10.1016/S1470-2045(08)70285-7

16. Bruix J, Cheng AL, Meinhardt G, Nakajima K, De Sanctis Y, Llovet J. Prognostic factors and predictors of sorafenib benefit in patients with hepatocellular carcinoma: analysis of two phase III studies. J Hepatol. 2017;67:999-1008. doi:10.1016/j.jhep.2017.06.026

17. Raoul JL, Adhoute X, Penaranda G, et al. Sorafenib: experience and better management of side effects improve overall survival in hepatocellular carcinoma patients: a real-life retrospective analysis. Liver Cancer. 2019;8(6):457-467. doi:10.1159/000497161

18. Marrero JA, Kudo M, Venook AP, et al. Observational registry of sorafenib use in clinical practice across Child-Pugh subgroups: the GIDEON study. J Hepatol. 2016;65(6):1140-1147. doi:10.1016/j. jhep.2016.07.020

19. Miller AA, Murry DJ, Owzar K, et al. Phase I and pharmacokinetic study of sorafenib in patients with hepatic or renal dysfunction: CALGB 60301. J Clin Oncol. 2009;27:1800-1805. doi:10.1200/ JCO.2008.20.0931

20. Parsa VK, Heilbrun L, Smith D, et al. Safety and efficacy of sorafenib therapy in patients with metastatic kidney cancer with impaired renal function. Genitourinary Cancer Symposium. Orlando, FL, USA; 2008. (Abstr 365).

21. Rey PM, Villavicencio H. Sorafenib: tolerance in patients on chronic hemodialysis: a single-center experience. Oncology. 2008;74:24 5-246. doi:10.1159/000151394

22. Lathia C, Lettieri J, Cihon F, Gallentine M, Radtke M, Sundaresan P. Lack of effect of ketoconazole-mediated CYP3A inhibition on sorafenib clinical pharmacokinetics. Cancer Chemother Pharmacol. 2006;57:685-692. doi:10.1007/s00280-005-0068-6

23. Deeken JF, Pantanowitz L, Dezube BJ. Targeted therapies to treat non-AIDS-defining cancers in patients with HIV on HAART therapy: treatment considerations and research outlook. Curr Opin Oncol. 2009;21:445-454. doi:10.1097/CCO.0b013e32832f3e04

24. Helis L, Ntinos N, Souftas V, et al. Complete response after sorafenib therapy for hepatocellular carcinoma in an HIV-HBV co infected patient: possible synergy with HAART? A case report. Med Oncol. 2011;28:S165-S168. doi:10.1007/s12032-010-9669-y

25. Perboni G, Costa P, Fibbia GC, et al. Sorafenib therapy for hepatocellular carcinoma in an HIV-HCV coinfected patient: a case report. Oncologist. 2010;15:142-145. doi:10.1634/theoncologist.2010-0010

26. Kim R, El-Gazzaz G, Tan A, et al. Safety and feasibility of using sorafenib in recurrent hepatocellular carcinoma after orthotopic liver transplantation. Oncology. 2010;79:62-66. doi:10.1159/000319548

27. Yoon DH, Ryoo BY, Ryu MH, et al. Sorafenib for re-current hepatocellular carcinoma after liver transplantation. Jpn J Clin Oncol. 2010;40:768-773. doi:10.1093/jjco/hyq055

28. Hajiev S, Allara E, Motedayen-Aval L, et al. Impact of age on sorafenib outcomes in hepatocellular carcinoma: an international cohort study. $\mathrm{Br}$ J Cancer. 2020;124:407-413. doi:10.1038/s41416-020-01116-9

29. Kudo M. Lenvatinib may drastically change the treatment landscape of hepatocellular carcinoma. Liver Cancer. 2018;7(1):1-19. doi:10. $1159 / 000487148$

30. Kimura T, Kato Y, Ozawa Y, et al. Immunomodulatory activity of lenvatinib contributes to antitumor activity in the Hepa1-6 hepatocellular carcinoma model. Cancer Sci. 2018;109(12):3993-4002. doi:10. 1111/cas.13806

31. Kato Y, Tabata K, Kimura T, et al. Lenvatinib plus anti-PD-1 antibody combination treatment activates $\mathrm{CD} 8+\mathrm{T}$ cells through reduction of tumor-associated macrophage and activation of the interferon pathway. PLoS One. 2019;14(2):e0212513. doi:10.1371/journal.pone. 0212513 
32. Takase N, Koma Y, Urakawa N, et al. NCAM- and FGF-2-mediated FGFR1 signaling in the tumor microenvironment of esophageal cancer regulates the survival and migration of tumor-associated macrophages and cancer cells. Cancer Lett. 2016;380(1):47-58. doi:10. 1016/j.canlet.2016.06.009

33. Kudo M, Finn RS, Qin S, et al. Lenvatinib versus sorafenib in first-line treatment of patients with unresectable hepatocellular carcinoma: a randomised Phase 3 non-inferiority trial. Lancet. 2018;391 (10126):1163-1173. doi:10.1016/S0140-6736(18)30207-1

34. Finn RS, Kudo M, Cheng A-L, et al. Final analysis of serum biomarkers in patients from the Phase 3 study of lenvatinib in unresectable hepatocellular carcinoma (REFLECT). Ann Oncol. 2018;29 (Suppl; abstr: 59PD):viii17-viii18. doi:10.1093/annonc/mdy269.057

35. Maruta S, Ogasawara S, Ooka Y, et al. Potential of lenvatinib for an expanded indication from the REFLECT trial in patients with advanced hepatocellular carcinoma. Liver Cancer. 2020;9(4):38 2-396. doi:10.1159/000507022

36. Sho T, Suda G, Ogawa K, et al. Early response and safety of lenvatinib for patients with advanced hepatocellular carcinoma in a real-world setting. JGH Open. 2019;4(1):54-60. doi:10.1002/ jgh3.12209

37. Sho T, Suda G, Ogawa K, et al. Lenvatinib in patients with unresectable hepatocellular carcinoma who do not meet the REFLECT trial eligibility criteria. Hepatol Res. 2020;50(8):966-977. doi:10.1111/ hepr.13511

38. Cheon J, Chon HJ, Bang Y, et al. Real-world efficacy and safety of lenvatinib in Korean patients with advanced hepatocellular carcinoma: a multicenter retrospective analysis. Liver Cancer. 2020;9 (5):613-624. doi:10.1159/000508901

39. Ogushi K, Chuma M, Uojima H, et al. Safety and efficacy of lenvatinib treatment in Child-Pugh A and B patients with unresectable hepatocellular carcinoma in clinical practice: a multicenter analysis. Clin Exp Gastroenterol. 2020;13:385-396. doi:10.2147/CEG.S256691

40. Tada T, Kumada T, Hiraoka A, et al. Safety and efficacy of lenvatinib in elderly patients with unresectable hepatocellular carcinoma: a multicenter analysis with propensity score matching. Hepatol Res. 2020;50(1):75-83. doi:10.1111/hepr.13427

41. Cheng A-L, Qin S, Ikeda M, et al. IMbrave150: efficacy and safety results from a ph III study evaluating atezolizumab (atezo) + bevacizumab (bev) vs sorafenib (Sor) as first treatment (tx) for patients (pts) with unresectable hepatocellular carcinoma (HCC). Ann Oncol. 2019;30(Supp19):LBA3. doi:10.1093/annonc/mdz446.002

42. Mazzaferro V, Regalia E, Doci R, et al. Liver transplantation for the treatment of small hepatocellular carcinomas in patients with cirrhosis. $N$ Engl J Med. 1996;334:693-700. doi:10.1056/NEJM 199603143341104

43. Kobayashi M, Kudo M, Izumi N, et al. Cost-effectiveness analysis of lenvatinib treatment for patients with unresectable hepatocellular carcinoma (uHCC) compared with sorafenib in Japan. $J$ Gastroenterol. 2019;54(6):558-570. doi:10.1007/s00535-019-01 554-0

44. Kim JJ, McFarlane T, Tully S, Wong WWL. Lenvatinib versus sorafenib as first-line treatment of unresectable hepatocellular carcinoma: a cost-utility analysis. Oncologist. 2020;25(3):e512-e519. doi:10.1634/theoncologist.2019-0501

45. Saiyed M, Byrnes J, Srivastava T, Scuffham P, Downes M. Costeffectiveness of lenvatinib compared with sorafenib for the first-line treatment of advanced hepatocellular carcinoma in Australia. Clin Drug Investig. 2020;40(12):1167-1176. doi:10.1007/s40261-02000983-7
46. Bruix J, Tak WY, Gasbarrini A, et al. Regorafenib as second-line therapy for intermediate or advanced hepatocellular carcinoma: multicentre, open-label, Phase II safety study. Eur J Cancer. 2013;49 (16):3412-3419. doi:10.1016/j.ejca.2013.05.028

47. Abou-Alfa GK, Meyer T, Cheng AL, et al. Cabozantinib in patients with advanced and progressing hepatocellular carcinoma. $N$ Engl J Med. 2018;379(1):54-63. doi:10.1056/NEJMoa1717002

48. Zhu AX, Kang YK, Yen CJ, et al. Ramucirumab after sorafenib in patients with advanced hepatocellular carcinoma and increased $\alpha$ fetoprotein concentrations (REACH-2): a randomised, double-blind, placebo-controlled, phase 3 trial. Lancet Oncol. 2019;20(2):282-296. doi:10.1016/S1470-2045(18)30937-9

49. Yau T, Kang YK, Kim TY, et al. Efficacy and safety of nivolumab plus ipilimumab in patients with advanced hepatocellular carcinoma previously treated with sorafenib: the CheckMate 040 randomized clinical trial. JAMA Oncol. 2020;6(11):e204564. doi:10.1001/ jamaoncol.2020.4564

50. Yau T, Park JW, Finn RS, et al. CheckMate 459: a randomized, multi-center phase 3 study of nivolumab (NIVO) vs sorafenib (SOR) as first-line (1L) treatment in patients (pts) with advanced hepatocellular carcinoma (aHCC). Ann Oncol. 2019;30(Suppl 5): v874-v875. doi:10.1093/annonc/mdz394.029

51. Finn RS, Ryoo BY, Merle P, et al. Pembrolizumab as second-line therapy in patients with advanced hepatocellular carcinoma in KEYNOTE-240: a randomized, double-blind, phase III trial. J Clin Oncol. 2020;38(3):193-202. doi:10.1200/JCO.19.01307

52. Pinter M, Scheiner B, Peck-Radosavljevic M. Immunotherapy for advanced hepatocellular carcinoma: a focus on special subgroups. Gut. 2020;gutjnl-2020-321702. doi:10.1136/gutjnl-2020-321702

53. Sonbol MB, Riaz IB, Naqvi SAA, et al. Systemic therapy and sequencing options in advanced hepatocellular carcinoma: a systematic review and network meta-analysis. JAMA Oncol. 2020;6(12):e204930. doi:10.1001/jamaoncol.2020.4930

54. Finn RS, Ikeda M, Zhu AX, et al. Phase Ib study of lenvatinib plus pembrolizumab in patients with unresectable hepatocellular carcinoma. J Clin Oncol. 2020;38(26):2960-2970. doi:10.1200/JCO. 20.00808

55. Cheng AL, Kang YK, Lin DY, et al. Sunitinib versus sorafenib in advanced hepatocellular cancer: results of a randomized phase III trial. J Clin Oncol. 2013;31(32):4067-4075. doi:10.1200/JCO.20 12.45 .8372

56. Johnson PJ, Qin S, Park JW, et al. Brivanib versus sorafenib as first-line therapy in patients with unresectable, advanced hepatocellular carcinoma: results from the randomized phase III BRISK-FL study. J Clin Oncol. 2013;31(28):3517-3524. doi:10.1200/JCO.2012. 48.4410

57. Zhu AX, Rosmorduc O, Evans TR, et al. SEARCH: a phase III, randomized, double-blind, placebo-controlled trial of sorafenib plus erlotinib in patients with advanced hepatocellular carcinoma. J Clin Oncol. 2015;33(6):559-566. doi:10.1200/JCO.2013.53.7746

58. Cainap C, Qin S, Huang WT, et al. Linifanib versus sorafenib in patients with advanced hepatocellular carcinoma: results of a randomized phase III trial. J Clin Oncol. 2015;33(2):172-179.

59. Abou-Alfa GK, Shi Q, Knox JJ, et al. Assessment of treatment with sorafenib plus doxorubicin vs sorafenib alone in patients with advanced hepatocellular carcinoma: phase 3 CALGB 80802 randomized clinical trial. JAMA Oncol. 2019;5(11):1582-1588. doi:10.10 01/jamaoncol.2019.2792 


\section{Publish your work in this journal}

The Journal of Hepatocellular Carcinoma is an international, peerreviewed, open access journal that offers a platform for the dissemination and study of clinical, translational and basic research findings in this rapidly developing field. Development in areas including, bu not limited to, epidemiology, vaccination, hepatitis therapy, pathology and molecular tumor classification and prognostication are all considered for publication. The manuscript management system is completely online and includes a very quick and fair peer-review system, which is all easy to use. Visit http://www.dovepress.com/ testimonials.php to read real quotes from published authors.

Submit your manuscript here: https://www.dovepress.com/journal-of-hepatocellular-carcinoma-journal 